\title{
Educación inclusiva para la intervención psicosocial en grupos de menores en situación de Vulnerabilidad.
}

Inclusive education for psychosocial intervention in groups of minors in a situation of vulnerability.

\author{
Reyna de los Ángeles Campa Álvarez \\ Universidad de Sonora (México) \\ reyna.campa@unison.mx \\ Blanca Aurelia Valenzuela \\ Universidad de Sonora (México) \\ blanca.valenzuela@unison.mx \\ Manuela Guillén Lúgigo \\ Universidad de Sonora (México) \\ blanca.valenzuela@unison.mx
}

Fecha recepción: 10/09/2019

Páginas 63-71

Fecha aceptación: 25/11/2019

\section{Resumen.}

El objetivo del presente estudio es detectar las estrategias inclusivas efectuadas por los profesores de educación básica para la intervención en estudiantes con una situación de vulnerabilidad Social. Para ello, se realizó una investigación con un enfoque cuantitativo, donde se aplicó el cuestionario- escala sobre la integración e inclusión de personas con necesidades educativas y diversas (Gento, 2008). La muestra se constituyó por 134 profesores pertenecientes de municipios de Hermosillo y Guaymas, Sonora (México). Los resultados indican que los profesores tienen una aceptación de que los alumnos poseen conocimientos de los que hay que partir, muestran afinidad hacia la diversidad humana; se muestra una necesidad de formación y capacitación apropiada para brindar la atención; se promueven los valores, existe democracia y las escuelas hacen valer el derecho a la inclusión. Por último, se evidencia el empleo de estrategias de trabajo colaborativo, promoción de valores y comunicación entre los miembros. La población vulnerable que se atiende con mayor frecuencia son: estudiantes con escasos recursos, necesidades educativas especiales, familias disfuncionales y problemas socioemocionales. Se concluye la importancia de una visión inclusiva, formación profesional y prácticas sociales para la efectividad de la educación inclusiva.

Palabras clave: educación inclusiva; vulnerabilidad social; rol del profesorado; estrategias inclusivas 


\section{Abstract.}

The objective of the present study is to detect the inclusive strategies carried out by elementary school teachers for intervention in students with a situation of Social vulnerability. For this, an investigation was carried out with a quantitative approach, where the questionnaire-scale on the integration and inclusion of people with educational and diverse needs was applied (Gento, 2008). The sample consisted of 134 professors belonging to the municipalities of Hermosillo and Guaymas, Sonora (Mexico). The results indicate that the professors have an acceptance that the students have knowledge from which they have to start, they show affinity towards human diversity; there is a need for appropriate education and training to provide care; values are promoted, democracy exists and schools assert the right to inclusion. Finally, the use of collaborative work strategies, promotion of values and communication between members is evident. The most frequent vulnerable population are: students with special educational needs, dysfunctional families and socio-emotional problems. The importance of an inclusive vision, professional training and social practices for the effectiveness of inclusive education is concluded.

Keywords: inclusive education; social vulnerability; teacher's role; inclusive strategies

\section{1.-Introducción.}

El sistema educativo actual en México enfrenta el reto de implementar políticas públicas que garanticen un aprendizaje de calidad a todos los estudiantes, independientemente de las características físicas, personales o sociales que posean. De acuerdo a la Secretaria de Educación Pública (SEP, 2017) la educación inclusiva se basa en la valoración de la diversidad, adaptando el sistema para responder de manera adecuada a las necesidades de todos y cada uno de los alumnos. En ese sentido, "se pretende asegurar la equidad y la calidad en la educación, considerando a todos los alumnos sin importar sus características, necesidades, intereses, capacidades, habilidades y estilos de aprendizaje" (p. 21).

La recomendación internacional insiste en la necesidad de dar un valor esencial a la educación inclusiva, como una acción de intervención y protectora de riesgos sociales; ya que la educación es algo inacabado, que empieza en los primeros años de vida y se mantienen a lo largo del tiempo (Casanova, 2011:95). Por lo cual, las escuelas tienen un papel clave, para ofrecer herramientas acordes a sus características particulares, de manera que todos y cada uno de los estudiantes de la comunidad se sientan acogidos, seguros y convencidos de que lograrán sus metas. La importancia de establecer un posicionamiento crítico permite visualizar, entender y realizar prácticas socio-culturales a través de procesos y escenarios que propicien la socialización, a partir del reconocimiento de las potencialidades humanas y como individuos con el pleno derecho a una vida digna.

Estudios realizados por Valenzuela, Guillén y Campa (2014), indican que los cambios que transcurre el ser humano durante la infancia y adolescencia tienen un impacto en 
el desarrollo psicosocial y los actores sociales que se involucran en este proceso, afectando significativamente la calidad de vida, a nivel psicológico, familiar, educativo y las relaciones sociales. Por lo cual, el desarrollo óptimo y sano del ser humano depende en gran medida de las interacciones que se forjan en las etapas tempranas del ciclo vital, de igual forma en las relaciones que establecen en el contexto social que se desenvuelve.

La educación inclusiva es una medida de atención a la diversidad que brinda un sentido de pertenencia y bienestar social para lograr un aprendizaje y rendimiento escolar adecuado acorde a sus necesidades. Echeita y Ainscow (2011), aluden al término de inclusión para referirse al énfasis que hace en aquellos grupos de alumnos y alumnas vulnerables, en riesgo de exclusión o rezago escolar, tales como individuos en situación de pobreza y marginación, migrantes, indígenas, e infantes que presentan discapacidad, siendo así una gran diversidad de población estudiantil.

Partiendo de lo anteriormente mencionado, el objetivo general del presente estudio es detectar las estrategias inclusivas efectuadas por los profesores de educación básica para la intervención en estudiantes con una situación de vulnerabilidad Social. Dentro de este contexto, el artículo se estructura partiendo del desarrollo de los conceptos de educación inclusiva, vulnerabilidad social e intervención psicosocial. Posteriormente se esboza la metodología de la investigación. Finalmente, se muestran los resultados y conclusiones del estudio.

\section{2.-Referentes conceptuales.}

\section{1.-Educación inclusiva.}

La educación inclusiva tiene una particular relevancia, no sólo vista como un derecho a la educación y al aprendizaje a lo largo de la vida, sino también como una vía para consolidar sociedades democráticas y justas, que brinden a todas las personas oportunidades de desarrollar su máximo potencial, con miras a un futuro sostenible y una existencia plena y digna. Por tanto, la educación inclusiva es un cambio global en sistema educativo, que afecta a todo el alumnado con un doble objetivo: conseguir el éxito de todos, sin excepciones, en la escuela; y luchar contra cualquier causa o razón de exclusión, segregación o discriminación.

Booth y Ainscow (2011) señalan que la educación inclusiva hace referencia a un proceso que pretende reducir las barreras del aprendizaje y la participación de todos los estudiantes, dicho proceso consta de tres dimensiones, 1) cultura, 2) política, y 3) práctica. Dentro de la dimensión cultural, se refiere a la comunidad educativa con valores y creencias compartidas. En la segunda dimensión, la inclusión es el impulsor del centro educativo y delimita las modalidades de apoyo para dar frente a la diversidad. En la tercera, las prácticas promueven las actividades de participación plena acorde a la cultura y capacidades del alumno. 


\section{2.-Vulnerabilidad social}

Los cambios sociales han generado que la vulnerabilidad se extienda y diversifica, afectando a un número creciente de niños, niñas, jóvenes, familias y a distintos sectores de la sociedad. Al referirse a la vulnerabilidad social se puede aludir a un proceso de exclusión acentuado (Ordoñez, 2018) que repercute en forma amplia el rezago social y bienestar de grupos poblacionales que no alcanzan los beneficios de inversión social.

La vulnerabilidad representa un estado de debilidad, ruptura de un equilibrio precario, que genera efectos negativos en el individuo o grupo de personas inmersos en estas situaciones. Uno de los rasgos distintivos es la incapacidad de actuar o de reaccionar a corto plazo; la vulnerabilidad no se limita a la falta de satisfacción de necesidades materiales, también incluye las conductas discriminatorias (Acevedo, Castro, Fernando y Trujillo, 2014).

Dichas problemáticas repercuten a nivel educativo; siendo un contexto social donde se configura por una gran diversidad humana. Siendo la población dominante en las escuelas estudiantes que se encuentran en riesgo o vulnerabilidad, es decir la diversidad educativa. Siendo la exclusión social uno de los principales problemas que afecta a grupos vulnerables que viven situaciones de desigualdad social, económica, educativa, etc.

\section{3.-Intervención psicosocial en las escuelas}

La atención a la diversidad vista desde la inclusión, es un proceso global que enmarca no sólo reconocer que existen estudiantes con forma de vida y características diferentes, sino además que estilos de aprendizaje, ritmos y diferencias individuales que hacen necesario que los actores sociales y profesionistas en el campo social proporcionen el apoyo con base a los principios de accesibilidad y equidad de oportunidades para el desarrollo social.

La inclusión social educativa es una medida para combatir a través de la educación las problemáticas que se presentan en nuestra sociedad, siendo considerados fundamentales para lograr mejoras en los estudiantes, buscando un cambio significativo en las diversas áreas que componen el panorama escolar, es decir, el área educativa, emocional y social, teniendo presente entre ellas: exclusión social, discriminación y poca accesibilidad de recursos; las cuales, no son controladas adecuadamente y pueden llevar a ciertas consecuencias (Molina, 2019).

La importancia de intervenir en la convivencia escolar, promover valores, generar redes de apoyo social y habilidades sociales permite construir una cultura inclusiva con respeto a la diversidad. A nivel individual se requiere trabajar variables como la estabilidad emocional, autoestima, sentido de pertenencia y fomentar metas en la vida. Por otra parte, trabajar con las actitudes y la práctica docente, así como la presencia de una cultura de diversidad, enmarcado en la igualdad de derecho y la promoción de 
valores; con el empleo de estrategias como el trabajo colaborativo con alumnos, fungen como ejes medulares en el proceso de inclusión (Campa y Contreras, 2018).

\section{3.-Metodología.}

La presente investigación fue bajo el enfoque cuantitativo de tipo descriptivocorrelacional. El diseño fue no experimental - transeccional, ya que las variables a medir no se ven manipuladas, basándose en la observación de fenómenos tal y como se dan en su contexto natural.

La muestra fue no probabilística intencional debido a que los participantes de la presenta investigación fue elegida a partir de ciertos criterios de inclusión. Los sujetos en total fueron 134 profesores de educación primaria pertenecientes de municipios de Hermosillo (64\%) y Guaymas (36\%); un $51 \%$ son mujeres y un $49 \%$ son hombres. El nivel educativo del profesorado, fue un $69 \%$ cuenta con estudios de licenciatura, $20 \%$ con el grado de maestría, $8 \%$, con especialidad y un 3\% con diplomado; los años de antigüedad docente oscilan entre 1 a 35 años, con una media entre 10-16 años. Los criterios de selección de los participantes fueron los siguientes: contexto educación primaria del sector público (ubicadas en sectores vulnerables), escuelas primarias con apoyo de los servicios y centros para la educación especial y la educación inclusiva en México y se consideraron todos los grados educativos de primero a sexto año.

Se aplicó el cuestionario-escala sobre la integración e inclusión de personas con necesidades educativas y diversas (Gento, 2008). Se retomaron las siguientes escalas: tipo de necesidad educativa, actitud de integración y estrategias de inclusión educativa. Se adaptaron los reactivos al contexto de estudio. La escala de evaluación utilizada es de tipo Likert, con opciones de respuesta: 1 (nunca), 2 (escasamente), 3 (frecuentemente), 4 (casi siempre) y 5 (siempre). La duración de la aplicación es aproximadamente de 15 -20 minutos. La alfa de Cronbach fue de .812 .

Para la aplicación del cuestionario- escala, se acudió a las escuelas primarias de los municipios de Hermosillo y Guaymas del estado de Sonora (México). Primeramente se solicitó la autorización con los respectivos directivos para tener el permiso para realizar el estudio, se presentó una carta explicativa con los fines de la investigación. Una vez obtenida la autorización, se prosiguió a reunir a los participantes para tener su consentimiento para participar. Una vez concluida la recolección se realizó su respectivo análisis, se utilizó la base estadística de datos SPSS versión 21.00 para realizar un análisis estadístico de fiabilidad, descriptivo y correlacional.

\section{4.-Resultados.}

En la gráfica 1, se muestran las medias de la escala de actitudes, los resultados indican medias altas en el reactivo lo maestros muestran afinidad hacia la diversidad humana (4.49) y en aceptación que todos los alumnos poseen conocimientos de los que hay que partir (4.22); sin embargo, se encontró una media baja de 2.80 que indica que 
escasamente los profesores consideran que los alumnos con diversidad pueden desarrollar sus propias potencialidades.

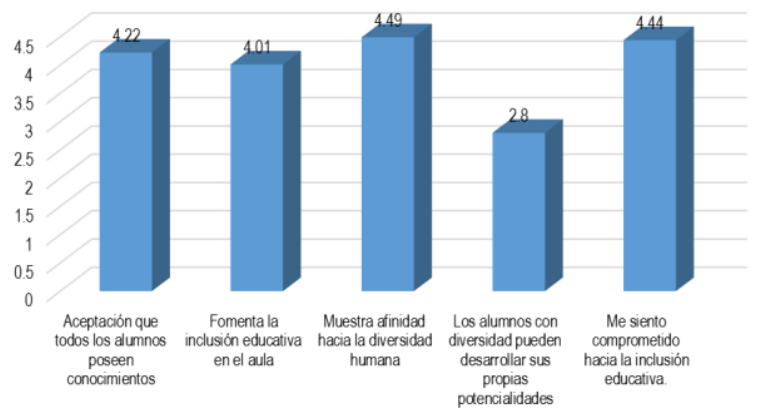

Gráfica 1. Medias de actitudes hacia la inclusión del profesorado de primaria.

Fuente: Elaboración propia (2019)

En la gráfica 2, se detecta los indicadores de educación inclusiva, se obtuvo media alta en el reactivo se aceptan a los alumnos con algún tipo de diversidad con 4.84 , se cuenta con una filosofía inclusiva (4.67); las medias bajas fueron en adecuaciones curriculares (3.43) y estrategias basadas en una visión común, lo cual indica limitantes en la inclusión de los grupos vulnerables en educación básica.

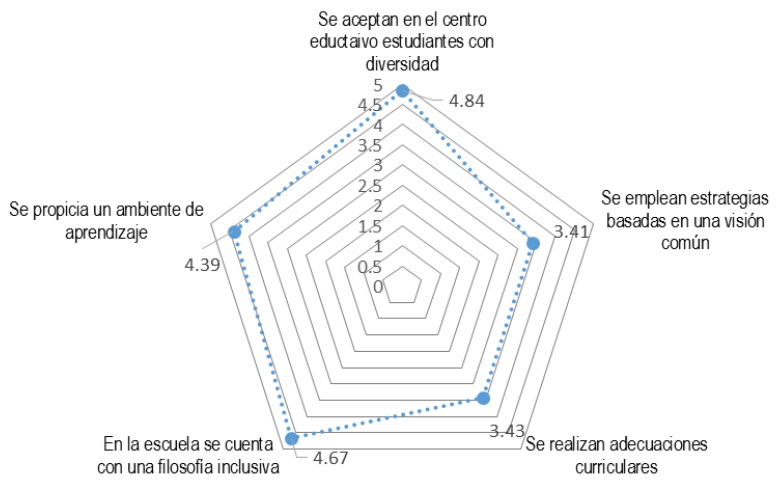

Gráfica 2. Medias de los indicadores de educación inclusiva.

Fuente: Elaboración propia (2019

Lo relacionado a la intervención psicosocial, se encontró que existen dificultades en la atención debido a que en las escuelas hay unos escases de campañas para promover la inclusión (3.42), se tiene una poca convivencia escolar entre los miembros (3.89) y la participación de los padres es reducida (3.98). Sin embargo, se logra resaltar un trato justo y equitativo con una media alta de 4.78 , la promoción de valores inclusivos en la escuela (4.85), comunicación entre los miembros (4.21). Tales resultados se muestran en la Gráfica 3. 


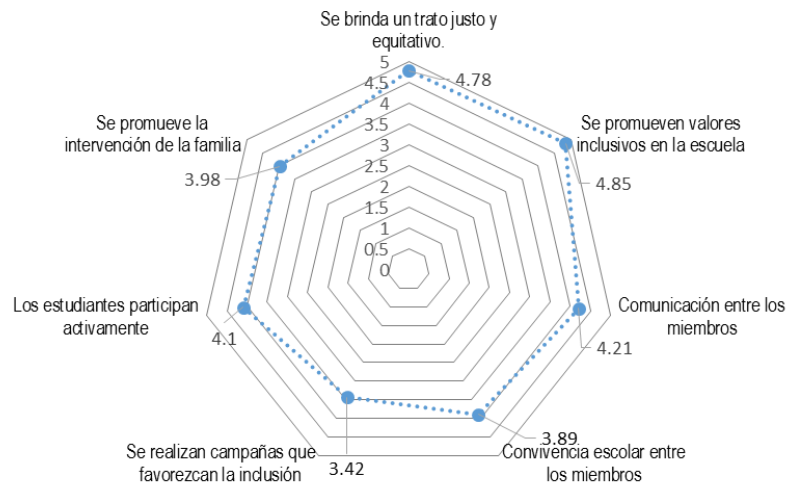

Gráfica 3. Medias de las estrategias de intervención psicosocial para la inclusión. Fuente: Elaboración propia (2019)

Por último, en la tabla 1 se muestran las correlaciones de Pearson siendo significativos entre las variables de estudio, pero principalmente a partir del tipo de vulnerabilidad que presente el estudiantes, se relaciona con la estrategias de intervención psicosocial $\left(.665^{\star *}\right)$, así como las actitudes de los profesores influyen con las estrategias de intervención $\left(.656^{* *}\right)$ y el uso de dichas estrategias se correlaciona altamente y significativamente con la educación inclusiva $\left(.670^{* *}\right)$.

Tabla 1. Correlaciones entre las variables de estudio

\begin{tabular}{lcccc}
\hline & $\begin{array}{c}\text { Tipo de } \\
\text { vulnerabilidad }\end{array}$ & Actitudes & $\begin{array}{c}\text { Educación } \\
\text { inclusiva }\end{array}$ & $\begin{array}{c}\text { Estrategias } \\
\text { de } \\
\text { intervención } \\
\text { psicosocial }\end{array}$ \\
\hline $\begin{array}{l}\text { Tipo de } \\
\text { vulnerabilidad }\end{array}$ & 1 & - & - & - \\
$\begin{array}{l}\text { Actitudes } \\
\text { Educación } \\
\text { inclusiva } \\
\begin{array}{l}\text { Estrategias de } \\
\text { intervención }\end{array}\end{array}$ & $.445^{*}$ & 1 & - & - \\
\hline
\end{tabular}

** La correlación es significativa al nivel 0,01 (bilateral).

*La correlación es significante al nivel 0,05 (bilateral).

\section{5.-Conclusión.}

En la sociedad actual con las transformaciones derivadas de la globalización, uno de los mayores retos en el sistema educativo es promover políticas y prácticas de inclusión que permitan alcanzar los aprendizajes básicos de la educación obligatoria a 
la diversidad de alumnado. En relación a las estrategias inclusivas se detecta que el aspecto cultual es un rubro que requiere atención, para el reconocimiento de la diversidad con un modelo educativo capaz de educar a una ciudadanía que comprenda, defienda y promueva las diferencias humanas como valor y derecho humano; es decir la cultura de la diversidad se basa en principios éticos y exige a la sociedad cambios en sus comportamientos con respecto a las personas segregadas.

Se evidencia con los resultados encontrados en la investigación que los participantes tienen las intenciones de incluir y atender; sin embargo son pocas las acciones y cursos que se realizan para llevarlo a cabo en la práctica cotidiana en las escuelas primarias. A la vez se refiere como demanda campañas sociales, en donde se trabaje en la concientización y sensibilización de la sociedad en general y la promoción de valores.

En este estudio, los participantes muestran una aceptación en los alumnos debido a que poseen conocimientos de los que hay que partir, a la vez afinidad hacia la diversidad humana; sin embargo, sólo aceptan diversidades de tipo motora, sociofamiliar y conductual, preferentemente leve y media; y poca aceptación a diversidad intelectual, auditiva y visual en sus diversos grados de afectación.

Los resultados indican los profesores tienen una aceptación de que los alumnos poseen conocimientos de los que hay que partir, muestran afinidad hacia la diversidad humana; necesidad de formación y capacitación apropiada para brindar la atención; se promueven los valores, existe democracia y las escuelas hacen valer el derecho a la inclusión, se emplean estrategias de trabajo colaborativo. La población vulnerable que se atiende con mayor frecuencia son: estudiantes en situación de pobreza, necesidades educativas especiales, familias disfuncionales. Se concluye la importancia de la visión inclusiva, formación profesional y prácticas sociales para la efectividad de la educación inclusiva.

Tomando en consideración que una educación inclusiva implica un sistema de enseñanza que atienda la diversidad humana de cualquier índole: discapacidad, necesidades educativas especiales, problemas socioemocionales, situación de pobreza, género, etnicidad, migración, entre otros. Ante la gama de estudiantes que se pueden encontrar inscritos en la educación básica obligatoria; se puede señalar que en las escuelas existe apertura para el ingreso de los estudiantes y una filosofía inclusiva; considerando las reformas educativas a favor de la inclusión como marco general; sin embargo resultan ineficaces al evidenciar la falta de adecuaciones a los programas educativos, las falta de campañas de promoción y la falta de organización escolar por parte del profesorado y directivos.

Por lo cual, la visión de las instituciones educativas demanda una apertura y flexibilidad transformadora, siendo el aula un escenario de intercambio e interacciones sociales, en donde la participación, la colaboración, la armonía y el compromiso de los actores educativos implicados es fundamental para la calidad educativa. Con base a lo antes planteado, algunas propuestas para posteriores investigaciones serian, involucrar a los 
estudiantes y padres de familia en el estudio, para conocer sus opiniones y sugerencias respecto a la educación inclusiva. Siendo la educación y la formación de los estudiantes un reto ante una sociedad cada vez más diversa y con desafíos para toda la comunidad educativa.

\section{6.-Referencias.}

Acevedo, J., Castro, L., Fernando, B., Trujillo, M. (2014). Problemática de los Grupos Vulnerables Visiones de la realidad. México: Nueva Era.

Booth, T., Ainscow, M. (2011). Índex para la inclusión. Guía para la evaluación y mejora de la educación inclusiva. Madrid: Consorcio Universitario para la Educación Inclusiva.

Campa, R., Contreras, C. (2018). Aspectos psicosociales asociados en el proceso de inclusión educativa durante la infancia intermedia. Infancias Imágenes, 7 (1), 9-24.

Casanova, M. (2011). Educación inclusiva: un modelo de futuro. Madrid: Wolters Kluwers.

Echeita, G., Ainscow, M. (2011). La educación inclusiva como derecho. Marco de referencia y pautas de acción para el desarrollo de una revolución pendiente. Tejuelo, 12, 26-46.

Molina, G. (2019). Valores inclusivos compartidos por la comunidad educativa del Instituto Montenegro (Quindío, Colombia): una aproximación desde los maestros. Revista Internacional de Apoyo a la Inclusión, Logopedia, Sociedad y Multiculturalidad, 5 (1), 86-104.

Ordoñez, G. (2018). Discriminación, pobreza y vulnerabilidad: los entresijos de la desigualdad social en México. Revista Región y Sociedad, 30 (71),

Secretaria de Educación Pública. (2017). Aprendizajes clave para la educación integral. México: SEP.

Sobrero, V. (2018). Hacia una docencia inclusiva en la Educación Superior: La investigación sobre la propia práctica docente como herramienta de transformación. Revista Chilena de Pediatría, 89 (1), 7-9

Valenzuela, B. A.; Guillén, M.; Campa, R. (2014). Recursos para la inclusión educativa en el contexto de educación primaria. Infancias Imágenes, 13 (2), 64-75. 